\title{
Magnetic Drug Targeting: Preclinical in Vivo Studies, Mathematical Modeling, and Extrapolation to Humans
}

\author{
Khuloud T. Al-Jamal, ${ }^{*}{ }^{\dagger}$ Jie Bai, ${ }^{\dagger}$ Julie Tzu-Wen Wang, ${ }^{\dagger}$ Andrea Protti, ${ }^{\S}$ Paul Southern, ${ }^{\prime}$ Lara Bogart, ${ }^{\|}$
} Hamed Heidari, ${ }^{\#}$ Xinjia Li, ${ }^{\dagger}$ Andrew Cakebread, ${ }^{\ddagger}$ Dan Asker, ${ }^{\dagger}$ Wafa T. Al-Jamal, ${ }^{\perp}$ Ajay Shah, ${ }^{\S}$ Sara Bals, Jane Sosabowski, ${ }^{\ddagger}$ and Quentin A. Pankhurst*,

${ }^{\dagger}$ Institute of Pharmaceutical Science, Faculty of Life Sciences \& Medicine and ${ }^{\ddagger}$ Analytical \& Environmental Sciences Division, Faculty of Life Sciences \& Medicine, King's College London, London SE1 9NH, U.K.

${ }^{\S}$ Cardiovascular Division, James Black Centre, King's College London British Heart Foundation Centre of Excellence, London, SE5 9NU, U.K.

"Healthcare Biomagnetics Laboratory, University College London, 21 Albemarle Street, London W1S 4BS, U.K.

${ }^{\perp}$ Dr. W.T. Al-Jamal, School of Pharmacy, University of East Anglia, Norwich Research Park, Norwich NR4 7TJ, U.K.

\#Electron Microscopy for Materials Research (EMAT), University of Antwerp, Groenenborgerlaan 171, B-2020 Antwerp, Belgium

${ }^{\nabla}$ Centre for Molecular Oncology, Bart's Cancer Institute, Queen Mary University of London, London EC1A 7BE, U.K.

\section{Supporting Information}

ABSTRACT: A sound theoretical rationale for the design of a magnetic nanocarrier capable of magnetic capture in vivo after intravenous administration could help elucidate the parameters necessary for in vivo magnetic tumor targeting. In this work, we utilized our long-circulating polymeric magnetic nanocarriers, encapsulating increasing amounts of superparamagnetic iron oxide nanoparticles (SPIONs) in a biocompatible oil carrier, to study the effects of SPION loading and of applied
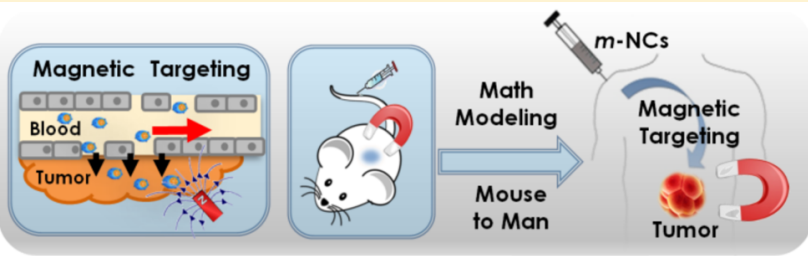
magnetic field strength on magnetic tumor targeting in CT26 tumor-bearing mice. Under controlled conditions, the in vivo magnetic targeting was quantified and found to be directly proportional to SPION loading and magnetic field strength. Highest SPION loading, however, resulted in a reduced blood circulation time and a plateauing of the magnetic targeting. Mathematical modeling was undertaken to compute the in vivo magnetic, viscoelastic, convective, and diffusive forces acting on the nanocapsules (NCs) in accordance with the Nacev-Shapiro construct, and this was then used to extrapolate to the expected behavior in humans. The model predicted that in the latter case, the NCs and magnetic forces applied here would have been sufficient to achieve successful targeting in humans. Lastly, an in vivo murine tumor growth delay study was performed using docetaxel (DTX)-encapsulated NCs. Magnetic targeting was found to offer enhanced therapeutic efficacy and improve mice survival compared to passive targeting at drug doses of ca. 5-8 mg of DTX/kg. This is, to our knowledge, the first study that truly bridges the gap between preclinical experiments and clinical translation in the field of magnetic drug targeting.

KEYWORDS: Polymeric nanocapsules, superparamagnetic iron oxide nanoparticles, SPECT imaging, cancer therapy, nanomedicine

uperparamagnetic iron oxide nanoparticles (SPIONs) have

$\checkmark$ been widely explored for pharmaceutical applications. ${ }^{1,2}$

Their superparamagnetic state ensures no remanent (permanent) moment in the absence of an applied field and obviates the risk of uncontrolled aggregation of the nanoparticles in the bloodstream or tissues. ${ }^{3}$ The directed transport of SPIONs in the bloodstream is dependent on a dynamic equilibrium between the magnetic and hydrodynamic forces acting on the SPIONs. ${ }^{4-8}$ As a result, the physical properties, including the field strength and gradients, the volumetric and magnetic properties (magnetization) of the particles, and the hydrodynamic parameters such as blood flow, hematocrit, viscosity, and SPION concentration in the blood, are all of importance. $^{1,6,9}$
Only one study investigating the optimal conditions on magnetic targeting has been reported so far. ${ }^{10}$ This study demonstrated that higher magnetic field strength offered better magnetic targeting. However, the conclusion was drawn from a custom-made magnetic setup and mainly determined on the basis of intra-arterial administration, which required invasive surgery. ${ }^{11}$ This was presumably due to the limited pharmacokinetic properties such as blood circulation half-life, organ biodistribution, and tumor extravasation ability. Thus, studying the key factors that possibly affect magnetic targeting efficacy is crucial.

Received: June 4, 2016

Revised: July 22, 2016

Published: August 19, 2016 
Table 1. Physicochemical Characterization of PEGylated NCs with Different SPION Loadings and Oil Cores Prepared by Single Emulsification-Solvent Evaporation Method

\begin{tabular}{|c|c|c|c|c|c|c|}
\hline formulation & $\begin{array}{l}\text { initial SPION } \\
\text { loading }^{a}\end{array}$ & $\underset{(\mathrm{nm})^{b, c}}{\operatorname{hydrodynamic} \text { size }} \pm \mathrm{SD}$ & $\mathrm{PDI} \pm \mathrm{SD}^{b, c}$ & $\zeta$ potential $\pm \mathrm{SD}$ & $\underset{\mathrm{SD}^{e, c}}{\operatorname{SPION}} \pm$ & $\begin{array}{l}\text { actual SPION } \\
\text { loading }\end{array}$ \\
\hline NC 1 & 0.00 & $203 \pm 4$ & $0.12 \pm 0.01$ & $-45 \pm 2$ & - & 0.00 \\
\hline NC 2 & 0.08 & $212 \pm 2$ & $0.17 \pm 0.02$ & $-38 \pm 1$ & $99 \pm 3$ & $0.08 \pm 0.01$ \\
\hline NC 3 & 0.38 & $218 \pm 4$ & $0.17 \pm 0.02$ & $-39 \pm 1$ & $99 \pm 2$ & $0.38 \pm 0.01$ \\
\hline $\mathrm{NC} 4$ & 1.85 & $218 \pm 3$ & $0.19 \pm 0.01$ & $-36 \pm 1$ & $95 \pm 3$ & $1.76 \pm 0.06$ \\
\hline NC 5 & 7.02 & $214 \pm 9$ & $0.19 \pm 0.02$ & $-31 \pm 1$ & $94 \pm 9$ & $6.60 \pm 0.63$ \\
\hline
\end{tabular}

${ }^{a}$ Values were expressed at w/w SPION-NC. Total NCs weight is calculated by the addition of polymer, lecithin, castor oil, SPION, and Tween 80 weights. Initial and final loading refers to SPION content before and after size-exclusion chromatography. ${ }^{b}$ Size was measured with dynamic light scattering and measured in deionized water. ${ }^{c}$ Results are expressed as mean $\pm \mathrm{SD}(n=3) .{ }^{d}$ Values were obtained with laser Doppler electrophoresis and measured in deionized water. ${ }^{e}$ Iron content was determined by ICP-MS.

a

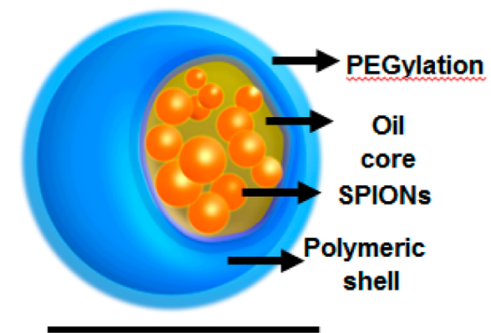

ca. $200 \mathrm{~nm}(\mathrm{NC} 1-5)$

C

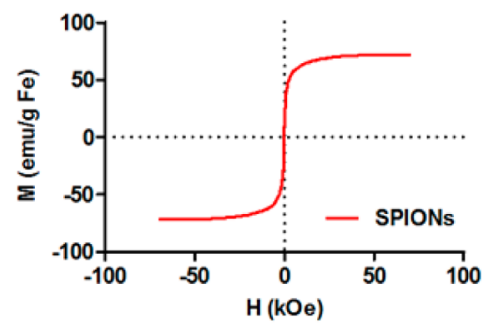

d

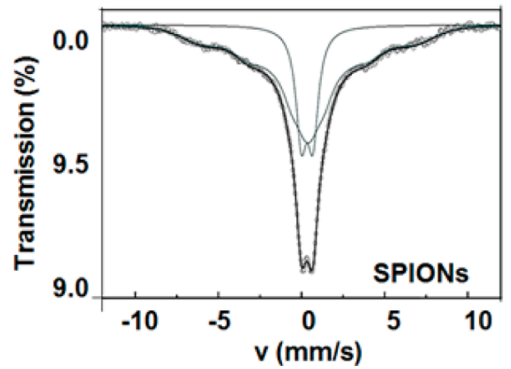

b
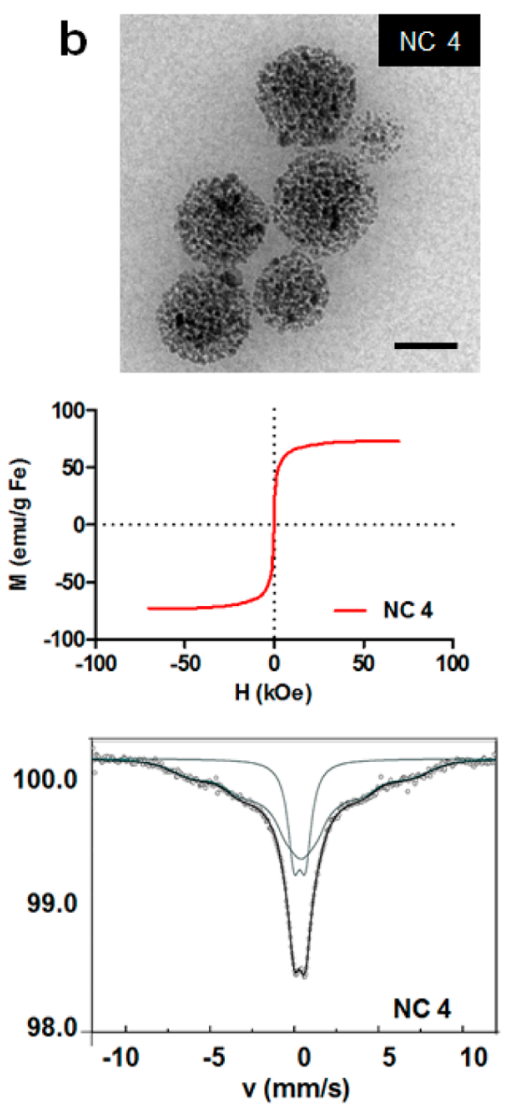

Figure 1. Physiochemical characterization of $m$-NCs. $m$-NCs were prepared by the single emulsification-solvent evaporation method. (a) Schematic illustration of $m$-NC structure, comprising a core-shell structure. (b) Cryo-TEM image of NC 4. (c) Magnetizations curve of the as-received SPIONs and a representative $m$-NC (NC 4) as a function of field, measured at $300 \mathrm{~K}$. The saturation magnetizations of both samples were ca. 72 $\mathrm{emu} / \mathrm{g}_{\mathrm{Fe}}$ (d) Transmission Mössbauer spectra of the as-received SPIONs and a representative $m$-NC (NC 4) recorded at room temperature. The mean isomer shift (spectral centroid) of the SPION and $m$-NCs was 0.38 and $0.39 \mathrm{~mm} / \mathrm{s}$, respectively, and is characteristic of a material composed largely of ferric ions.

In this work, we used oil-cored polymeric magnetic nanocapsules ( $m$-NCs) with tunable SPION loadings and prolonged blood circulation time ${ }^{12}$ to better understand the factors influencing magnetic targeting after intravenous injections in vivo. The high SPION loading eliminated the need for using highly magnetized nanoparticles. The oil core facilitated high hydrophobic drug loading compared to that of the polymer-coated SPIONs reported in the literature. ${ }^{13,14}$ Our aim was to assess the key factors influencing magnetic targeting efficacy, including SPION loadings in $m$-NC and the remotely applied magnetic force magnitude. To translate the results from mouse to human, mathematical modeling was undertaken to compute the in vivo magnetic, viscoelastic, convective, and diffusive forces acting on the $m$-NCs in accordance with the Nacev-Shapiro construct. ${ }^{15}$ The predicted magnetic targeting behavior in mouse vasculature was compared with the observed murine data and then extrapolated to the expected behavior in humans. Docetaxel (DTX) was used as a model hydrophobic anticancer drug to test the therapeutic efficacy of $m$-NCs in the presence or absence of magnetic targeting.

The use of SPIONs in the biomedical field is advantageous given the superparamagnetic state. Their small size $(<30 \mathrm{~nm})$ also allows them to extravasate through the leaky blood vasculature of tumors. ${ }^{16,17}$ However, through the $2000 \mathrm{~s}$ and 

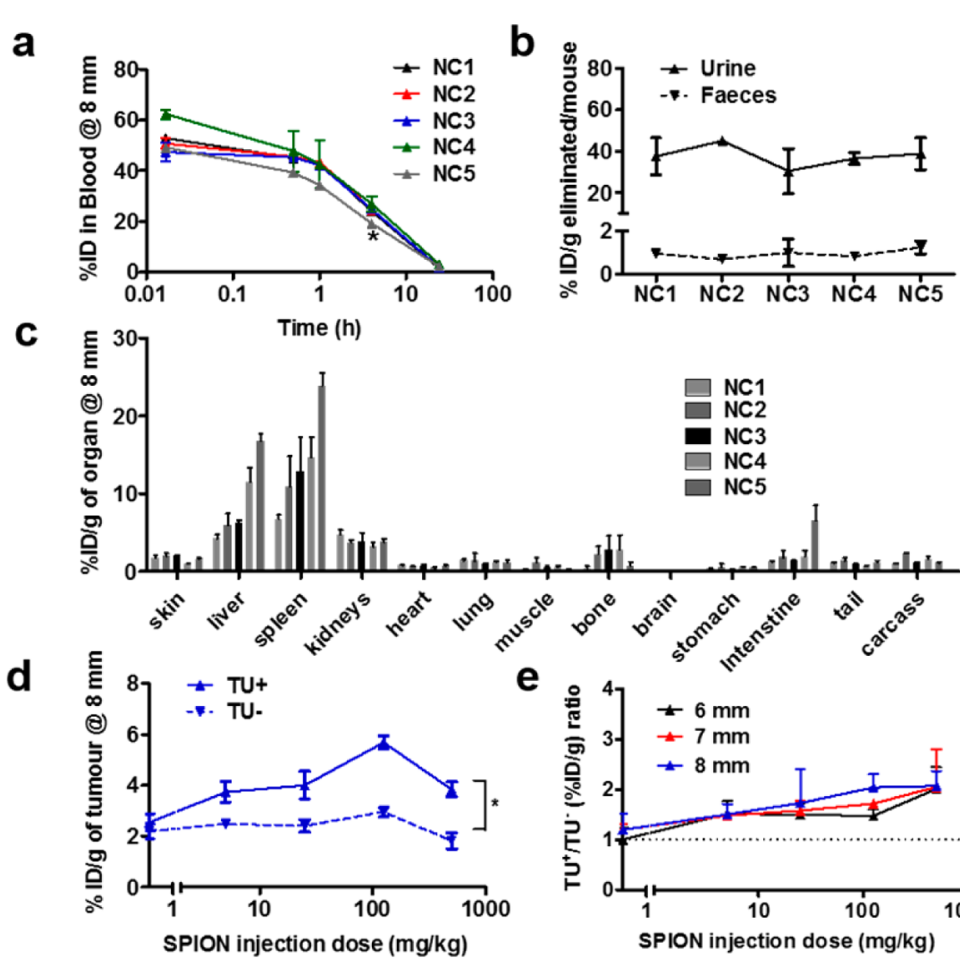

$\mathbf{f}$
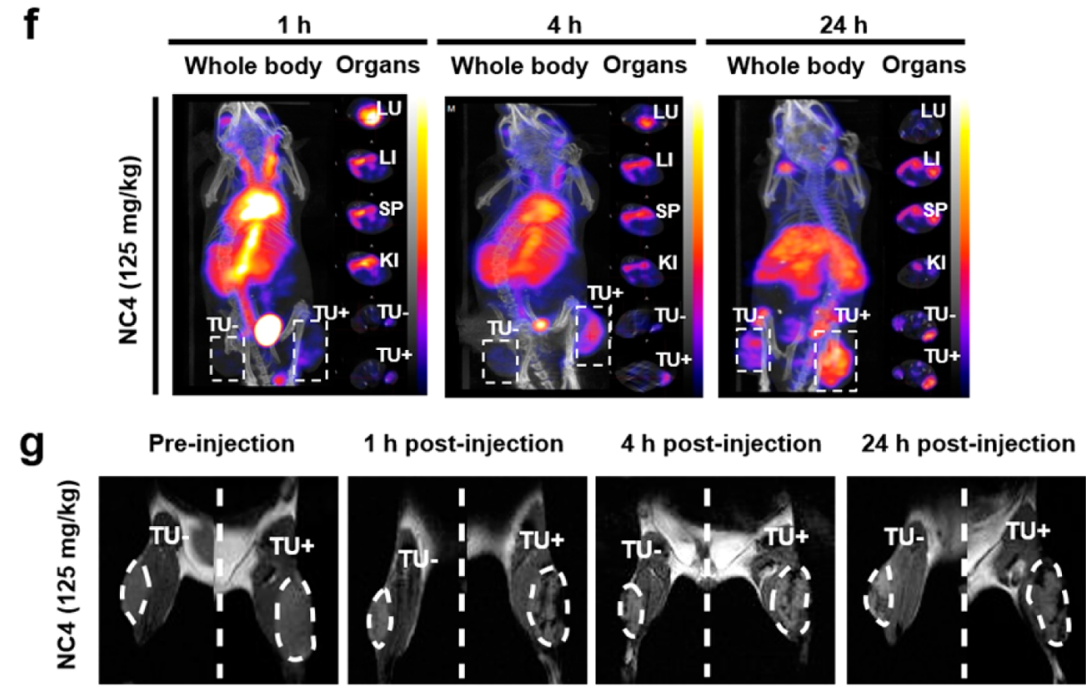

Figure 2. In vivo magnetic targeting assessment of $m$-NC- ${ }^{111}$ In with increasing amounts of SPION in CT26 tumor-bearing BALB/c mice under the influence of an $8 \mathrm{~mm}$ diameter magnet $(0.43 \mathrm{~T})$. Mice were iv injected with indium-111 labeled NC $1-5$. A permanent magnet $(0.43 \mathrm{~T}, 8 \mathrm{~mm}$ in diameter) was applied at one tumor site ( $\mathrm{TU}+)$ for $1 \mathrm{~h}$, and organs were excised at $24 \mathrm{~h}$ post-injection. (a) Blood clearance profiles. (b) Excretion profiles. (c) Organ biodistribution profile. (d) Tumor accumulation profiles. (e) Magnetic targeting efficacy. (f) In vivo single-photon emission computed tomography-computed tomography (SPECT-CT) imaging of $m$-NC $4-^{-111} \mathrm{In}$. (g) In vivo $\mathrm{T}_{2}$-weighted $\mathrm{MR}$ imaging of $m$-NC $4-{ }^{111} \mathrm{In}$. Cross-sections in (f) were from lung (LU), liver (LI), spleen (SP), kidney (KI), nonmagnetically targeted tumor (TU-), and magnetically targeted tumor $(\mathrm{TU}+)$ at equivalent time points. Tumors in (f) and (g) are marked in dashed lines. Results are expressed as \% ID/g of organ as mean \pm SEM $(n=3)$. One-way ANOVA was performed using IBM SPSS Statistics software followed by Tukey's multiple comparison test $(*, p<0.05 ; * *, p<$ $0.01)$.

2010s, SPION-based magnetic drug targeting has been the subject of intense debate, with concerns focusing on the minimal magnetic forces generated from the small magnetic cores and the limited blood circulation times of the SPIONs used. $^{10,11,18,19}$ To address these issues, we developed a polymeric oil-cored $m$-NC system prepared with increasing amounts of SPION ( $0 \%$ to ca. $7 \%$ w/w SPION-NC, namely NC 1-5) with PEGylated poly(lactic-co-glycolic acid) (PLGAPEG). ${ }^{12}$ The hydrodynamic sizes were in the range of 203-218 $\mathrm{nm}$, and no significant changes were observed over the 3 month storage period (Table 1 and Figure S1). A schematic morphological illustration of an $m$-NC is shown in Figure 1a. $m$-NCs are expected to have a core-shell structure with SPIONs entrapped within the oil core and surrounded by a polymeric shell and PEGylated outer layer. Figure $1 \mathrm{~b}$ shows the $2 \mathrm{D}$ projections of $m$-NCs imaged by low-dose cryo-transmission electron microscopy (cryo-TEM), from which the image strongly suggests that a high number of SPIONs (electron-dense nanoparticles) are well-confined within the oil core. The superparamagnetic properties were not compromised 
as confirmed by the superconducting quantum interference device (SQUID) and Mössbauer spectroscopy analysis (Figure $1 c, d)$. This design potentially offered an increase in the apparent magnetic forces acting on the nanocapsules as a whole compared to SPIONs, as magnetic force is directly proportional to the cumulative iron oxide nanoparticle volume within the $m$ NC core.

Fundamental work of biodistribution profiles and tumor accumulation was conducted to understand the key factors in achieving efficacious magnetic targeting. NC $1-5$ were radiolabeled with indium-111 (Table S1), as we reported previously. ${ }^{12}$ Magnetic targeting efficacy was assessed by calculating the fold increase in percentage injected dose per gram of tumor tissue (\%ID/g of tumor) upon application of magnetic field.

To explore the effect of SPION loading on magnetic targeting efficiency, mice were injected with comparable NC doses (312.5 mg of polymer $/ \mathrm{kg}$ ) with increased loadings of SPIONs from 0 to ca. $7 \% \mathrm{w} / \mathrm{w}$ in $m$-NCs. This was achieved by preparing $m$-NCs with different initial SPION loadings (NC 1$5)$. The effect of SPION loading on blood circulation, excretion, organ biodistribution, and tumor accumulation was examined using the formulated $m$-NC- ${ }^{111}$ In with magnetic field of $0.43 \mathrm{~T}$. Blood circulation profiles are shown in Figure 2a. All $m$-NCs displayed prolonged blood circulation time with approximately $40 \%$ and $20 \%$ remaining in the blood after 1 and $4 \mathrm{~h}$, respectively. $m$-NCs with the highest SPION loading (ca. 7\%, NC 5- ${ }^{111} \mathrm{In}$ ), however, showed significantly lower blood circulation by $4 \mathrm{~h}$ post-injection $(p<0.05)$. It is unclear why NC with the highest SPION loading circulated shorter than the other formulations, but one may hypothesize that the iron overloading induced NC instability, more notably under the reasonably high shear forces in vivo. The gradual reduction in $\zeta$ potential values, as SPION loading increases, is another parameter that one may want to consider; NC 5 exhibited the lowest $\zeta$ potential among all formulation.

Negligible radioactivity was found in feces (0-2\% ID/ mouse), and nearly $40 \% \mathrm{ID} /$ mouse was detected in urine over the first $24 \mathrm{~h}$ post-injection period (Figure $2 \mathrm{~b}$ ) for all $\mathrm{m}$-NCs. Instant thin-layer chromatography (iTLC) confirmed that only $5.3-9.3 \%$ of the excreted radioactivity was in the form of ${ }^{111} \mathrm{In}$ DTPA-EDTA chelates indicating that PLGA $_{18 \mathrm{KDa}}-\mathrm{PEG}_{3.5 \mathrm{KDa}}{ }^{-}$ DTPA was excreted intact. Organ biodistribution of NC 1$5-{ }^{111} \mathrm{In}$ was assessed at $24 \mathrm{~h}$ post-injection (Figure 2c). The highest uptake was observed in spleen followed by liver and kidney tissue. Increasing SPION loadings led to a gradual increase in liver and spleen uptake and became statistically significant at $7 \% \mathrm{w} / \mathrm{w}(p<0.01)$. Such results explain the reduction in blood circulation time of $m$-NCs of highest SPION loading.

Uptake in tumors exposed to magnetic field (TU+) or that remained unexposed (TU-) was quantified and is shown in Figure 2 d. Significantly higher \% ID/g of tumor was observed in TU+ compared to TU- in NC $2-5$ but at a different extent $(p<0.05)$. No significant differences in $\% \mathrm{ID} / \mathrm{g}$ of tumor (TU-) were observed with $m$-NCs at increasing loadings of SPION, except for NC 5 with the SPION loading (ca. 7\%), which showed the lowest but significant uptake. This was expected as a result of reduced blood circulation time and increased liver and spleen uptake. Despite lower \% ID/g of tumor obtained for NC 5, its enhancement in tumor uptake upon magnetic targeting was still significant and comparable to that of NC 4 (Figure 2e). The effect of SPION loading on in vivo magnetic targeting efficacy was also confirmed by singlephoton emission computed tomography-computed tomography (SPECT-CT) imaging (Figure $2 \mathrm{f}$ and Figure S2). Interestingly, signals could be seen in tumors exposed to magnet ( $\mathrm{TU}+$ ) at $1 \mathrm{~h}$ post-injection, possibly due to magnetic targeting, while signals in TU- only started to be obvious at $4 \mathrm{~h}$ post-injection due to the passive EPR effect. Tumor accumulation of $m$-NCs was enhanced over time in both tumors for all formulations. Higher intensity was seen in TU+ compared to TU- at all imaging time points studied. Because the $m$-NCs were also a good magnetic resonance (MR) contrast agent, ${ }^{12} \mathrm{MR}$ imaging was also performed to confirm the tumor uptake of SPIONs with increasing SPION loadings (Figures $2 \mathrm{~g}$ and S3). Similar to SPECT-CT imaging, more hypo-intense signals (darkening) were seen in TU+ than TUin mice injected with $\mathrm{NC} 2-5$, indicating higher SPION accumulation in tumors when the magnetic field was applied. The intensities from MRI were not compared between $m$-NCs due to different initial SPION injection doses (indicated in the Supplementary Methods section).

The effect of magnetic field on tumor uptake was studied using three magnets with different magnetic field strength/ gradients $(0.43,0.41$, and $0.30 \mathrm{~T})$ and dimensions $(8,7$, and 6 $\mathrm{mm}$ in diameter, respectively). Organ biodistribution for NC 1-5 was not altered when different magnetic field strength and gradients were applied to the tumor (Figures S4 and S5, respectively). However, at lower magnetic field strength and gradients $(0.30$ and $0.41 \mathrm{~T})$, no magnetic targeting was obtained in mice injected with NC 2 (the lowest SPION loading) (Figures S4c and S5c). This is most probably because the low magnetic field provided insufficient magnetic forces on $m$-NCs under these conditions. On the contrary, when the strongest magnetic field $(0.43 \mathrm{~T})$ was applied, tumor uptake increased linearly when SPION doses increase from 0 to 125 $\mathrm{mg}$ SPION $/ \mathrm{kg}$. The results suggest that magnetic targeting is still possible at lower magnetic field, but $m$-NCs of higher SPION loadings are required for this instance. As a result, high SPION loading and magnetic field strength and gradients were therefore proven to be the determining factors to achieve efficient magnetic targeting experimentally. To the best of our knowledge, this is the first study to investigate the influence of the magnetic field, at increasing SPION doses, on magnetic targeting efficacy in vivo.

Another concern in the field of magnetic targeting research is the lack of translation of mouse and rat experimental data to human clinical designs. We, therefore, have applied magnetic force calculation to the in vivo experimental conditions (viz., a $10 \mathrm{~mm}$ diameter tumor adjacent to the center of the circular face of the $\mathrm{NdFeB}$ actuating magnet) to quantify the magnitude of the magnetic forces experienced by $m$-NCs as they pass through the tumor (see the Supplementary Methods section and Figures S6-S13). In the case of NC 4 with the $8 \mathrm{~mm}$ diameter magnet, these forces range from ca. 0.3 to $6.5 \mathrm{fN}$ per $m$-NC, with a median force (i.e., the force experienced by an $m$ NC containing 43 SPIONs per $m$-NC (the center of the polydispersity range) at the center of the tumor $(5 \mathrm{~mm}$ from the face of the magnet)) of ca. $1.9 \mathrm{fN}$ per $m$-NC. This quantification allows us to extrapolate from the observed behavior in the mouse model to the expected behavior in humans by reference to the finite element modeling work of Nacev et al. ${ }^{20,21}$

The Nacev model is particularly useful here because it is formulated in terms of three dimensionless physical parameters 
(the magnetic Richardson number $\Psi$, the mass Peclet number $\mathrm{Pe}$, and the reduced Renkin diffusion coefficient $D$ ) that may be evaluated for both preclinical and clinical situations. This simulates the competition between the applied magnetic forces, blood convection, and diffusion and therefore reveals the magnetic behaviors of $m$-NCs under any given physiological conditions. Figure 3a shows the calculated Nacev behavior for the mouse model for the case of NC 4 with the $8 \mathrm{~mm}$ diameter magnet (further details on the calculations are given in the Supplementary Methods section). The magnetic Richardson
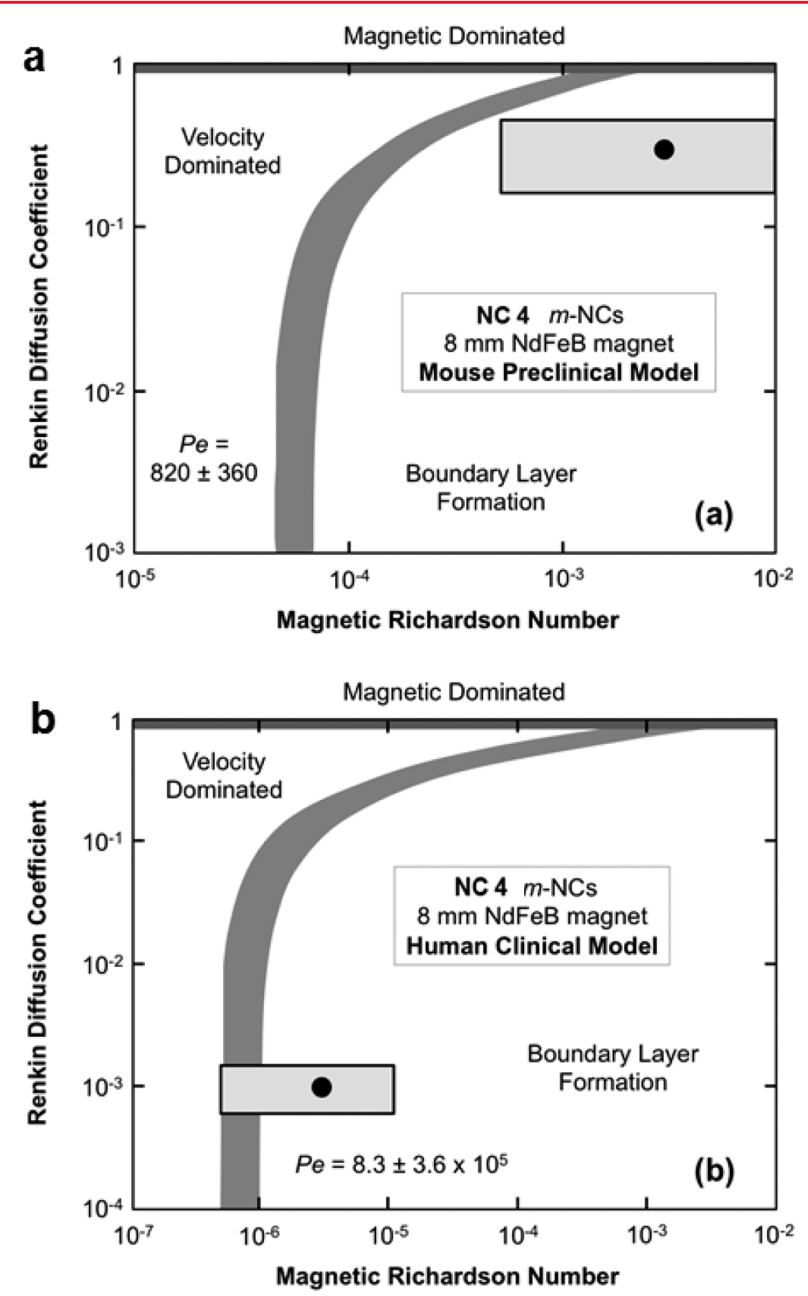

Figure 3. Calculated magnetic targeting efficacy of NC 4 with the 8 $\mathrm{mm}$ diameter $\mathrm{NdFeB}$ magnet for both the CT26 mouse model and a projected human clinical model. In accordance with the Nacev magnetic targeting model, the three characteristic parameters that govern the magnetic capture behavior of magnetic entities in blood vessels (the magnetic Richardson number $\Psi$, the mass Peclet number $\mathrm{Pe}$, and the reduced Renkin diffusion coefficient $D$ ) were calculated for $\mathrm{NC} 4$ with the $8 \mathrm{~mm}$ diameter $\mathrm{NdFeB}$ magnet. Results are shown for (a) the CT26 mouse model of this work and (b) a postulated human clinical model of superficial solid tumors accessed by $5 \mathrm{~mm}$ diameter blood vessels. Variations due to the polydispersity of the number of SPIONs per nanocapsule and the different vascular pathways that the $m$-NCs might take through the tumor were considered; hence, the symbol marks the most-probable ( $\Psi$ and $D$ ) combination, and the shaded box denotes the range of \pm 1 standard deviation in those parameters. The variation in $\mathrm{Pe}$ is evident in the breadth of the intermediate zone separating the regions of positive capture ("boundary-layer formation") and of limited capture ("velocitydominated"). number $\Psi$, the mass Peclet number Pe, and the reduced Renkin diffusion coefficient $D$ triad in this case were estimated to be $\Psi$ $=3.0_{-2.5}^{+8.0} \times 10^{-3}, \mathrm{Pe}=820 \pm 360$, and $D=0.30 \pm 0.13$, which places the predicted magnetic targeting behavior firmly in the region of positive capture, i.e., in the "boundary-layer formation" region. ${ }^{20,21}$ The other formulation that positioned in this region was NC 5 (ca. $7 \% \mathrm{w} / \mathrm{w}$ ) (Figure S14). $m$-NCs with lower SPION loadings, such as NC 2 (ca. $0.08 \% \mathrm{w} / \mathrm{w}$ ), were mostly located in the velocity-dominated region or the phase-transition layer, indicating that the magnetic forces are weak compared with the blood flow forces; ${ }^{15}$ thus, NC 2 are likely to be washed out of the blood vessels, which agreed with our experimental data.

These results made us confident in scaling our mouse experimental results to the virtual human body by fitting the magnetic force calculations into the Nacev model while using human blood hydrodynamic parameters. We adopted the blood and blood vessel parameters in humans from the Lübbe et al. Phase I clinical study, ${ }^{22}$ as reported by Nacev et al. ${ }^{20,21}$ and listed in the Supporting Information. Magnetic targeting tends to be more difficult to achieve in humans because human blood velocity and vessel diameters are at least 3 orders of magnitude greater than those observed in both mice and rats. The tissuemembrane diffusion coefficient is also ca. 3-fold higher. Figure $3 \mathrm{~b}$ shows the predicted Nacev behavior in humans, again for the case of NC 4 and the $8 \mathrm{~mm}$ diameter magnet. In this case, the behavior-governing environment parameters were set to values appropriate for ca. $5 \mathrm{~mm}$ skin-to-target distance, as defined in an earlier human clinical trial of magnetic targeting of near-to-skin solid tumors. ${ }^{23}$ The calculated triad values here were $\Psi=3.0_{-2.5}^{+8.0} \times 10^{-6}, \mathrm{Pe}=8.3+3.6 \times 10^{5}$, and $\mathrm{D}=1.0+0.4$ $\times 10^{-3}$. It is apparent from Figure $3 \mathrm{~b}$ that the predicted magnetic targeting behavior in the human blood vessels remains, for the most part, in the region of positive capture.

When the experimental and mathematical data are taken together, it is obvious that the pharmacokinetic profile of $\mathrm{m}$ NCs also plays a crucial role in determining efficacy of magnetic targeting, apart from the magnetic forces. For example, NC 4 showed higher blood circulation time than NC 5, which agreed with TU+ uptake values (NC 4:5.7\%ID/g and NC 5:3.8\%ID/g, $p<0.05)$ and therefore could deliver more drug to a tumor. As a result, the high SPION loading acts as a trade-off between strong magnetic forces and ideal pharmacokinetic properties in a magnetic nanocarrier design. This suggests that for this particular formulation, the optimal SPION loading is ca. $2 \%$, above which the magnetic targeting effect is counterbalanced by shortened blood circulation time due to clearance by the reticular endothelial system (RES); hence, no further improvement in magnetic targeting could be seen.

In this study, the $m$-NC size of $>200 \mathrm{~nm}$ was found to perform better in magnetic targeting than the small-sized SPION reported in the literature, more likely due to the enhancement in the magnetic force acting per $m$-NC as a result of their larger diameter. It is worth noting that this is not the ideal size with which to exploit the EPR effect in mouse tumors and, in particular, also in tumors in human patients. We, however, showed that smaller-sized PLGA-based nanoparticles $($ size $\sim 150 \mathrm{~nm})$, prepared without the oil core, were also able to benefit from magnetic targeting of the tumors (data not shown). It is also worth mentioning that the magnetic field gradient used in this study is relatively low, and stronger magnets can be used if smaller magnetic carriers are to be used. 
Table 2. Physicochemical Characterization of PEGylated DTX Encapsulated NCs Prepared by Single Emulsification-Solvent Evaporation Method

\begin{tabular}{|c|c|c|c|c|c|}
\hline formulation & initial DTX loading $^{a}$ & hydrodynamic size $\pm \mathrm{SD}(\mathrm{nm})^{b, c}$ & $\mathrm{PDI} \pm \mathrm{SD}^{b, c}$ & $\zeta$ potential $\pm \mathrm{SD}(\mathrm{mV})^{d, c}$ & $\mathrm{DTX} \mathrm{EE} \% \pm \mathrm{SD}^{e, c}$ \\
\hline NC-DTX & $4 \%$ & $215 \pm 13$ & $0.19 \pm 0.03$ & $-52 \pm 2$ & $83 \pm 2$ \\
\hline$m$-NC-DTX & $4 \%$ & $215 \pm 9$ & $0.17 \pm 0.02$ & $-41 \pm 1$ & $85 \pm 1$ \\
\hline$m$-NC-DTX & $10 \%$ & $217 \pm 1$ & $0.20 \pm 0.01$ & $-50 \pm 2$ & $75 \pm 4$ \\
\hline
\end{tabular}

$a_{\%} / \mathrm{w}$ DTX-polymer. ${ }^{b}$ Size was measured with dynamic light scattering and measured in deionized water. ${ }^{c}$ Results are expressed as mean \pm SD $(n$ $=3) .{ }^{d}$ Values were obtained with laser Doppler electrophoresis and measured in deionized water. ${ }^{e}$ DTX content was determined by reverse-phase high-performance liquid chromatography.

a

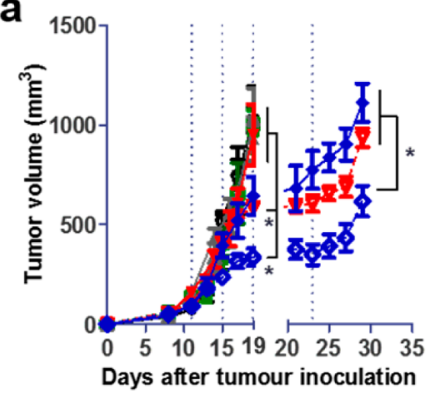

b

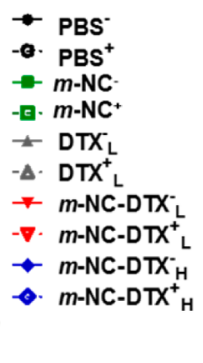

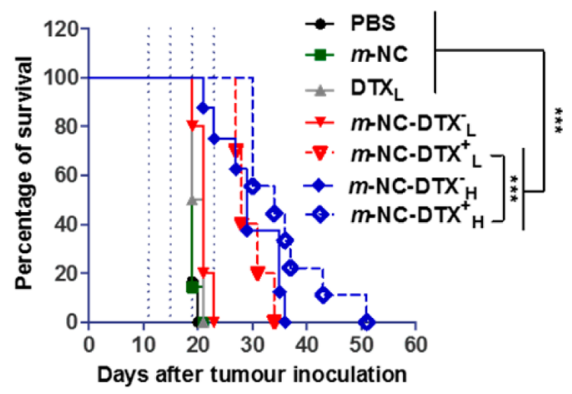

TU+

C
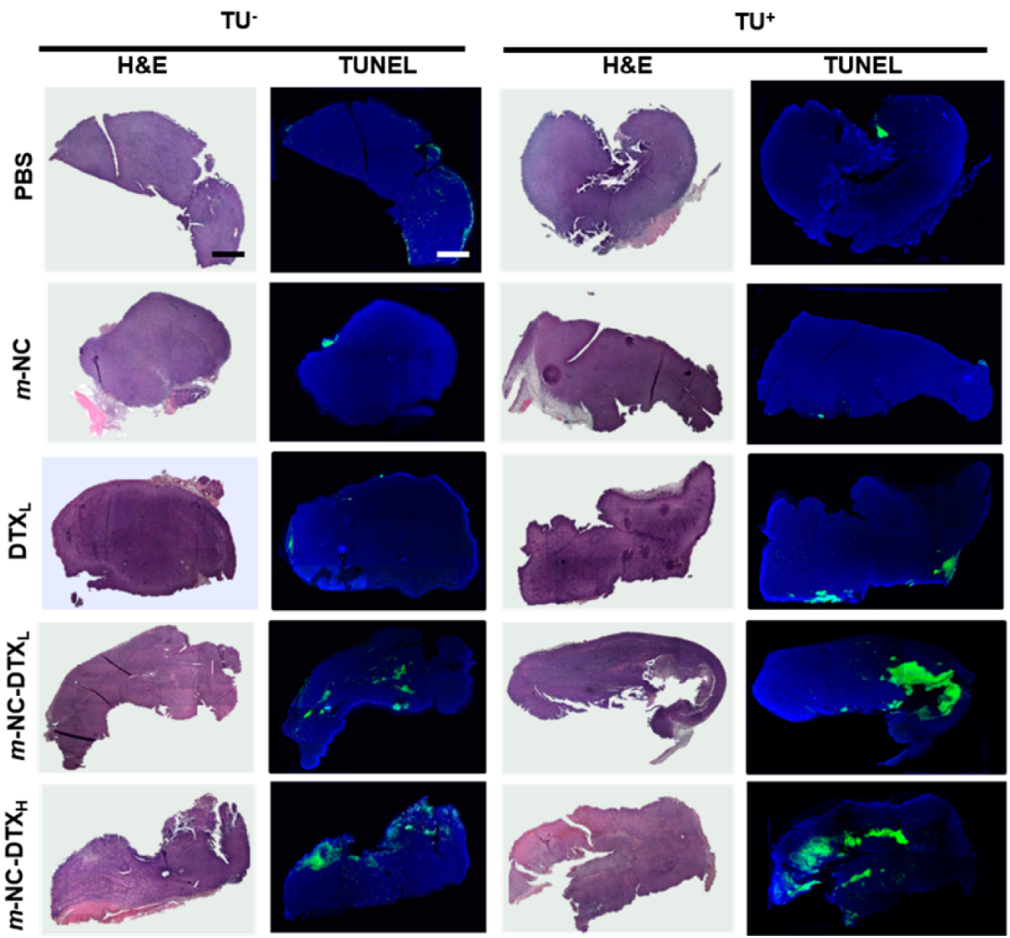

Figure 4. In vivo therapeutic effect in BALB/c mice after multiple injections of $m$-NC-DTX in combination with magnetic targeting in the CT26 colon cancer model. Mice were subjected to multiple injections of the therapy, which were carried out every 4 days from day 11 . A permanent magnet $(0.43 \mathrm{~T}, 8 \mathrm{~mm}$ in diameter) was applied at one tumor site (TU+) in all magnetically targeted groups for $1 \mathrm{~h}$ after each injection. (a), Tumor growth curve of CT26 tumors. Dashed lines indicate four injection dates. Results are shown as mean \pm SEM $(n=6-10)$. (b) Survival analysis of tumor-bearing mice. Mice were culled when the largest tumor diameter reached $15 \mathrm{~mm}$ in length. (c) Hematoxylin and eosin staining (H\&E) and terminal deoxynucleotidyl transferase dUTP nick-end labeling (TUNEL) staining of tumors after multiple injections. TUNEL and DAPI were counterstained to identify apoptotic (green) cells from the total cell population (blue nuclei) in TUNEL assay. Scale bars are $1000 \mu \mathrm{m}$. One-way ANOVA was performed for tumor growth curve using SPSS IBM 20 followed by Tukey's multiple comparison test, and a log-rank test was performed for the survival curve using Graph Pad (*, $p<0.05$; **, $p<0.005)$.

It is crucial to point out that in this study the $m$-NC were prepared with different amounts of SPION loading, which may influence their size and $\zeta$ potential and can indirectly affect the organ biodistribution and magnetic tumor targeting profiles. Despite the gradual reduction in $\zeta$ potential as SPION loading increased, our data showed that there was no difference in $m$ -
NC size or organ biodistribution profile nor blood circulation time, except for NC 5, which coincidentally exhibited the least negative $\zeta$ potential. It is likely that the discrepancy between the modeling results of NC 5 and the in vivo magnetic tumor targeting is related to their dramatically reduced blood circulation time and their reduced $\zeta$ potential compared to 
those of other formulations. One way to overcome this problem could be to prepare $m$-NC of a fixed SPION loading but then alter the number of $m$-NCs injected per mouse to alter the SPION dose administered. This approach may have its own limitations, such as different degrees of RES saturation, making this approach not perfect either.

The only in vivo study reported so far is by Chertok et al., ${ }^{10}$ which examined the magnetic retention of MNPs under different magnetic field gradients and topography in intracerebral 9L-glioma bearing rats. When magnetic field strength increased from 0.15 to $0.35 \mathrm{~T}$, a 2.1 -fold increase in iron content in tumor tissue was observed. The topography of magnetic field also affects the accumulation of MNPs in tumors. The conventional permanent magnets or electromagnets usually generate a relatively uniform magnetic field gradient over a broad range. As a result, all vasculatures, especially tumor arteries, tend to be exposed to high magnetic forces, thereby causing vascular embolism when MNPs are injected intraarterially. ${ }^{8}$ In this study, we report that magnetic targeting can be achieved with intravenously injected magnetic nanocarriers. Our study, however, did not offer a solution to targeting deep or metastatic tumors and was only limited to superficial tumors. More innovative solutions are still to be found for magnetically targeting deep tumors.

To test the therapeutic outcome of $m$-NCs with magnetic targeting, a cancer therapy study was carried out using docetaxel (DTX) as a model hydrophobic drug in a CT26 colon cancer model. DTX suffers from poor water solubility (10-20 $\mu \mathrm{g} / \mathrm{L})$, which hampers its ability to be administered systemically. ${ }^{24}$ The only commercial formulation available now is Taxotere DTX (Sanofi-Aventis), but it is reported to have several hypersensitivity reactions and incompatibility with common peripheral venous catheters, ${ }^{24,25}$ so there is ongoing substantial effort to develop better DTX formulations. In this study, DTX was coencapsulated in the SPION-loaded oil-cored polymeric $m$-NCs with the purpose of effectively delivering high doses of drugs to cancers with the magnetic targeting strategy. ${ }^{14}$ The physicochemical properties of DTX loaded $m$ NCs ( $m$-NC-DTX) were not significantly affected (Table 2) and $m$-NC also demonstrated sustained drug-release profiles (Figure S15a). An efficient cell-killing effect of $m$-NC-DTX against the CT26 cell line was observed in the in vitro cytotoxicity study (Figure S15b). Therefore, optimal magnetic targeting conditions were employed in the therapy study, i.e., NC 4 (ca. 2\%) with the $8 \mathrm{~mm}$ diameter magnet. To test the efficacy of magnetic targeting, two different doses of DTX were employed: (i) $5 \mathrm{mg} / \mathrm{kg}$ as the low $(\mathrm{L})$ dose $\left(m-\mathrm{NC}_{-} \mathrm{DTX}_{\mathrm{L}}\right)$ and (ii) $8 \mathrm{mg} / \mathrm{kg}$ as the high $(\mathrm{H})$ dose $\left(m-\mathrm{NC}-\mathrm{DTX}_{\mathrm{H}}\right)$. Mice were either exposed to the magnet, e.g., $\mathrm{DTX}^{+}$, or remained unexposed, e.g., DTX ${ }^{-}$.

Tumor growth curves responding to different treatments are shown in Figure 4a. Mice injected with DTX $_{\mathrm{H}}$ showed signs of significant body weight loss after two or three injections and were sacrificed before the completion of the treatment. Hence, the results are not included. In the other groups, no differences in tumor growth curves were observed between $\mathrm{DTX}^{ \pm}{ }_{\mathrm{L}} \mathrm{PBS}^{ \pm}$, and $m-\mathrm{NC}^{ \pm}$(no-drug) treatments, and mice reached the terminal stage on day 19 with tumor volumes of $\sim 1000 \mathrm{~mm}^{3}$. However, tumor growth delay was observed by day 19 in groups receiving $m$-NC-DTX $\mathrm{H}_{\mathrm{H}}$ with or without magnetic field ( $337 \pm 43$ and $645 \pm 95 \mathrm{~mm}^{3}$, respectively). Interestingly, only the magnetically targeted groups, $m$-NC-DTX ${ }_{\mathrm{H}}$ and $m$-NC$\mathrm{DTX}_{\mathrm{L}}^{+}\left(586 \pm 34 \mathrm{~mm}^{3}\right)$, showed significantly smaller tumor volume compared to that of the control treatments by day 19 $(p<0.05)$. No tumor growth inhibition was found in the $m$ NC-DTX $^{-}$group $\left(951 \pm 152 \mathrm{~mm}^{3}\right.$ by day 19$)$. By day 29 , mice receiving $m-\mathrm{NC}^{-\mathrm{DTX}^{+}}{ }_{\mathrm{H}}$ showed significantly delayed tumor growth compared to $m-\mathrm{NC}_{-} \mathrm{DTX}^{-}{ }_{\mathrm{H}}$ or $m-\mathrm{NC}^{-\mathrm{DTX}^{+}}{ }_{\mathrm{L}}(p$ $<0.05)$.

To our knowledge, only one study reported the use of DTX against CT26 tumors. This was in a metastasis model using PLGA microparticles at a dose of $8 \mathrm{mg} / \mathrm{kg}^{26}$ PLGA microparticles prolonged the median survival times of mice by 10 days. This was similar to the results obtained in this study at the same administration dose when no magnet was applied and at a lower dose with magnet, whereas the survival rate increased from 19 to 34 days $(p<0.005)$ when the magnetic targeting strategy was applied (Figure 4b). Results confirmed our hypothesis that improvement in cancer therapy in vivo can be achieved and lower drug doses can be given systemically without compromising the therapeutic efficacy when optimal magnetic targeting conditions are employed.

Tumors were also terminal deoxynucleotidyl transferase dUTP nick-end labeling (TUNEL)-stained to determine the degree of apoptosis after DTX therapy and histologically examined using hematoxylin and eosin $(\mathrm{H} \& \mathrm{E})$ staining (Figure $4 c$, right and left panels, respectively). Tumors from PBS, empty $m$-NCs, and free DTX groups exhibited few green patches (apoptotic cells), which were observed mainly at the tumor periphery. Expectedly, larger apoptotic areas were found in $m$-NC-DTX ( $\mathrm{L}$ and $\mathrm{H}$ ) treated tumors, more significantly with the ones subjected to magnetic field. H\&E staining of fixed tissue sections showed no major signs of necrosis in the major organs of mice in all experimental groups except for $\mathrm{DTX}_{\mathrm{H}}$, in which spleen angiectasis and hemorrhage associated with fibrosis were found (Figure S16a). These abnormalities were not seen at lower doses $(5 \mathrm{mg} / \mathrm{kg})$ or the same dose in $m$-NC formulation $\left(m-\mathrm{NC}-\mathrm{DTX}_{\mathrm{H}}\right)$. The inflammation-mediated toxicity (TNF- $\alpha$ levels) and liver function (liver biomarker level) were also assessed after the systemic administration of $m$-NCs or $m$-NC-DTX. No obvious increase was observed in both tests compared to the results from negative controls (Figures S16b and S17). Overall, these results suggest the biocompatibility of the formulated $m$-NCs in vivo under the conditions tested, and the $m$-NC formulation significantly reduced the systemic toxicity of DTX.

This study was designed to enable better understanding of the factors crucial for magnetic drug targeting in mice following intravenous administration and, furthermore, to extrapolate those preclinical findings to humans. Mathematical modeling was undertaken to compute the in vivo magnetic, viscoelastic, convective, and diffusive forces acting on $m$-NCs in accordance with the Nacev-Shapiro construct, and parameters were determined for which the theory successfully predicted the observed murine data. These parameters were then used to extrapolate to the expected behavior in humans, both generally and for the previously reported case conditions of a clinical trial on magnetic targeting to superficial solid tumors. The model predicted that in the latter case, the $m$-NCs and magnetic forces applied here would have been sufficient to achieve successful targeting in humans. Lastly, an in vivo murine tumor growth delay study was performed using the $2 \% \mathrm{w} / \mathrm{w}$ SPION-NC nanocapsules with docetaxel (DTX), an anticancer drug, incorporated into the oil core. Magnetic targeting was found to offer enhanced therapeutic efficacy and improved mice 
survival compared to the results from passive targeting at a drug dose of ca. 5-8 mg of DTX $/ \mathrm{kg}$.

This work presented here bridges the gap between preclinical experiments and clinical translation in the field of magnetic drug targeting. Conceptual advance in both the fundamental scientific understanding of the technology and its performance have been made during this work. This is exampled by the ability to calculate the magnetic force exerted on each nanocapsule and links such values to the preclinical targeting data. Such values were used to extrapolate magnetic targeting efficiency from mouse to human. The method can be used to predict magnetic targeting efficiency in preclinical or clinical subjects once information on the diameters of SPION and the magnetic carrier, the tumor's position in relation to the magnet, and blood vessels characteristics become available. Conversely, the method allows us to design magnetic field setups of characteristics optimal for achieving the most-efficient magnetic targeting in vivo for a bespoken magnetic nanocarrier.

\section{ASSOCIATED CONTENT}

\section{S Supporting Information}

The Supporting Information is available free of charge on the ACS Publications website at DOI: 10.1021/acs.nanolett.6b02261.

Additional details on materials and reagents, size and $\zeta$ potential measurements, determination of SPION encapsulation efficiency in $m$-NCs, superconducting quantum interference device magnetometry, Mössbauer spectroscopy, shelf stability of $m$-NCs, determination of DTX content by reverse-phase high-performance liquid chromatography, in vitro DTX release studies by dialysis, in vitro cytotoxicity studies by MTT assay, radio labeling of $m$-NCs and serum stability studies, animal studies and tumor inoculation, magnetic targeting setup in vivo, live small animal SPECT-CT imaging studies, quantitative organ biodistribution studies by $\gamma$ scintigraphy, estimation of the magnetic forces acting on the $m$-NCs, Nacev model calculations, hematoxylin and eosin staining of tissue sections, TUNEL staining of tumor sections, evaluation of TNF- $\alpha$ level after systemic administration of $m-\mathrm{NC}$ in vivo, statistical analysis, docetaxel loading in $m$-NCs and \%EE, DTX release from $m$-NC-DTX, in vitro cytotoxicity studies in CT26 colon cancer cells, and TNF- $\alpha$ level and systemic toxicity examination in mice subjected to DTX therapy in combination with magnetic targeting. A table showing radio-labeling stability of PEGylated NCs with different SPION loadings by the single emulsification-solvent evaporation method. Figures showing shelf-life stability of PEGylated $m$-NCs with different SPION loadings; in vivo SPECT-CT imaging of $m$-NC- ${ }^{111} \mathrm{In}$; in vivo $\mathrm{T}_{2}$-weighted MR studies of $m$ $\mathrm{NC}$; organ biodistribution, blood clearance, and tumor accumulation profiles of $m-\mathrm{NC}-{ }^{111} \mathrm{In}$; representative finite element modeling of the magnetic field magnitude $\mathrm{B}$ plots; finite element modeling estimates; a representative cryo-TEM micrograph of an individual magnetic nanocapsule; distribution of SPION diameters measured from cryo-TEM micrographs of magnetic nanocapsules from the NC4 sample; actuation forces calculated for NC 1-5 samples for the case of the 6-8 $\mathrm{mm}$ diameter cylindrical $\mathrm{NdFeB}$ permanent magnet; comparison of the median actuation forces calculated for $\mathrm{NC} 1-5$ for all three cylindrical $\mathrm{NdFeB}$ permanent magnets; calculated magnetic targeting efficacy of NC 2-5 $m$-NCs with the $8 \mathrm{~mm}$ diameter NdFeB magnet for CT26 mouse model; in vitro release profile and cytotoxicity of $m$-NC-DTX in CT26 murine colon carcinoma cells; histological examination of major organs; and systemic toxicity in CT26 BALB/c tumor-bearing mice after multiple injection of $m$-NC-DTX and DTX. (PDF)

\section{AUTHOR INFORMATION}

\section{Corresponding Authors}

*E-mail: khuloud.al-jamal@kcl.ac.uk. *E-mail: q.pankhurst@ucl.ac.uk.

\section{Notes}

The authors declare no competing financial interest.

\section{ACKNOWLEDGMENTS}

J.B. acknowledges funding from the King's-China Scholarship Council (CSC). Funding from the Biotechnology and Biological Sciences Research Council (BB/J008656/1), Worldwide Cancer Research (12-1054), and EU FP7-ITN Marie-Curie Network programme RADDEL (290023) is acknowledged. Q.P. is grateful to A. Nacev (Weinberg Medical Physics, Rockville, MD) and to B. Shapiro (University of Maryland, College Park, MD) for their useful advice during the preparation of this manuscript.

\section{REFERENCES}

(1) Pankhurst, Q. A.; Connolly, J.; Jones, S.; Dobson, J. J. Phys. D: Appl. Phys. 2003, 36, R167.

(2) Scialabba, C.; Licciardi, M.; Mauro, N.; Rocco, F.; Ceruti, M.; Giammona, G. Eur. J. Pharm. Biopharm. 2014, 88, 695-705.

(3) Williams, P. S.; Carpino, F.; Zborowski, M. Mol. Pharmaceutics 2009, 6, 1290-1306.

(4) Grief, A. D.; Richardson, G. J. Magn. Magn. Mater. 2005, 293, $455-463$

(5) Goya, G.; Grazu, V.; Ibarra, M. Curr. Nanosci. 2008, 4, 1-16.

(6) Cherry, E. M.; Maxim, P. G.; Eaton, J. K. Med. Phys. 2010, 37, 175.

(7) Voltairas, P.; Fotiadis, D.; Michalis, L. J. Biomech. 2002, 35, 813821.

(8) Chertok, B.; David, A. E.; Huang, Y.; Yang, V. C. J. Controlled Release 2007, 122, 315-323.

(9) Babincova, M.; Babinec, P. Biomed. Pap. 2009, 153, 243-250.

(10) Chertok, B.; David, A. E.; Yang, V. C. J. Controlled Release 2011, 155, 393-399.

(11) Cole, A. J.; David, A. E.; Wang, J.; Galbán, C. J.; Yang, V. C. Biomaterials 2011, 32, 6291-6301.

(12) Bai, J.; Wang, J. T. W.; Rubio, N.; Protti, A.; Heidari, H.; ElGogary, R. I.; Southern, P.; Al-Jamal, W. T.; Sosabowski, J.; Shah, A.; Bals, S.; Pankhurst, Q. A.; Al-Jamal, K. T. Theranostics 2016, 6, 342356.

(13) El-Gogary, R. I.; Rubio Carrero, N.; Wang, J. T.-W.; Al-Jamal, W. T.; Bourgognon, M.; Kafa, H.; Naeem, M.; Klippstein, R.; Abbate, V.; Leroux, F.; et al. ACS Nano 2014, 8, 1384-1401.

(14) Klippstein, R.; Wang, J. T. W.; El-Gogary, R. I.; Bai, J.; Mustafa, F.; Rubio, N.; Bansal, S.; Al-Jamal, W. T.; Al-Jamal, K. T. Small 2015, $11,4704-4722$.

(15) Nacev, A.; Beni, C.; Bruno, O.; Shapiro, B. Nanomedicine 2010, $5,1459-1466$.

(16) Cole, A. J.; Yang, V. C.; David, A. E. Trends Biotechnol. 2011, 29, 323-332.

(17) Estelrich, J.; Escribano, E.; Queralt, J.; Busquets, M. A. Int. J. Mol. Sci. 2015, 16, 8070-8101. 
(18) Rudge, S.; Kurtz, T.; Vessely, C.; Catterall, L.; Williamson, D. Biomaterials 2000, 21, 1411-1420.

(19) Jurgons, R.; Seliger, C.; Hilpert, A.; Trahms, L.; Odenbach, S.; Alexiou, C. J. Phys.: Condens. Matter 2006, 18, S2893.

(20) Nacev, A.; Beni, C.; Bruno, O.; Shapiro, B. Nanomedicine (London, U. K.) 2010, 5, 1459-66.

(21) Nacev, A.; Beni, C.; Bruno, O.; Shapiro, B. J. Magn. Magn. Mater. 2011, 323, 651-668.

(22) Lübbe, A. S.; Bergemann, C.; Riess, H.; Schriever, F.; Reichardt, P.; Possinger, K.; Matthias, M.; Dörken, B.; Herrmann, F.; Gürtler, R. Cancer Res. 1996, 56, 4686-4693.

(23) Lubbe, A. S.; Bergemann, C.; Riess, H.; Schriever, F.; Reichardt, P.; Possinger, K.; Matthias, M.; Dorken, B.; Herrmann, F.; Gurtler, R.; Hohenberger, P.; Haas, N.; Sohr, R.; Sander, B.; Lemke, A. J.; Ohlendorf, D.; Huhnt, W.; Huhn, D. Cancer Res. 1996, 56, 4686-93. (24) Youm, I.; Yang, X. Y.; Murowchick, J. B.; Youan, B.-B. C. Nanoscale Res. Lett. 2011, 6, 1-12.

(25) Mei, L.; Zhang, Y.; Zheng, Y.; Tian, G.; Song, C.; Yang, D.; Chen, H.; Sun, H.; Tian, Y.; Liu, K.; et al. Nanoscale Res. Lett. 2009, 4, $1530-1539$.

(26) Fan, R.; Wang, Y.; Han, B.; Luo, Y.; Zhou, L.; Peng, X.; Wu, M.; Zheng, Y.; Guo, G. Int. J. Biol. Macromol. 2014, 69, 100-107. 